\title{
Preoperative use of anti-tumor necrosis factor therapy in Crohn's disease: promises and pitfalls
}

\author{
Paulo Gustavo Kotze ${ }^{1}$, Subrata Ghosh ${ }^{2}$, Willem A. Bemelman ${ }^{3}$, Remo Panaccione ${ }^{4}$ \\ ${ }^{1}$ Colorectal Surgery Unit, Cajuru University Hospital, Catholic University of Paraná, Curitiba, Brazil, ${ }^{2}$ Institute of Translational Medicine, \\ University of Birmingham, Edgbaston, Birmingham, UK, ${ }^{3}$ Department of Surgery, Academic Medical Center, Amsterdam, The Netherlands, \\ ${ }^{4}$ Inflammatory Bowel Disease Unit, University of Calgary, Alberta, Canada
}

Recent advances in medical and surgical therapy were achieved during the last two decades in the management of Crohn's disease (CD). Anti-tumor necrosis factor (anti-TNF) agents are widely used worldwide. However, a significant proportion of patients still need surgical resections. The impact of previous exposure to these agents on the perioperative and postoperative outcomes is still controversial. In this critical review, we aimed to position the strategy of intentional preoperative use of antiTNF agents in the management of CD. The indications and contraindications for this strategy are detailed, and despite scarce evidence, the possible advantages and disadvantages of the intentional use of anti-TNF agents before abdominal surgery in CD are discussed. (Intest Res 2017;15:160-165)

Key Words: Crohn disease; Tumor necrosis factor-alpha; Preoperative care

\section{INTRODUCTION}

In the management of $\mathrm{CD}$, different therapeutic strategies aimed at disease control are available. During the last two decades, significant advances in medical therapy, mainly based on clinical experiences with biological agents, were achieved. ${ }^{1}$ These new agents also contributed to reducing the surgical rates; however, surgery is still needed in a significant proportion of patients. The surgical therapy for CD has also evolved, mainly owing to the growing expertise in minimally invasive techniques, such as multiport and single-port laparoscopy, and the development of innovative surgical devices that can make these new techniques feasible. ${ }^{2}$

Important advances were observed, not only in the technology itself but also in strategies and care pathways, in patients with CD undergoing surgery. This can be represented by the development of enhanced recovery protocols and the individualized definition of the proper timing for the surgical procedure, mainly based on the relation of surgical outcomes with perioperative medical therapy, mostly after the biological era. ${ }^{3,4}$

Currently, abdominal surgery is indicated for CD; however, a considerable number of patients have previous exposure to biological therapy, and there is still controversy about

Received December 26, 2016. Revised January 19, 2017. Accepted January 19, 2017. Published online March 21, 2017

Correspondence to Paulo Gustavo Kotze, Colorectal Surgery Unit, Cajuru University Hospital, Catholic University of Paraná, Rua Mauá 682, Curitiba 80030-200, Brazil. Tel: +55-41-3022-5500, Fax: +55-41-3243-0033,E-mail: pgkotze@hotmail.com

Financial support: None. Conflict of interest: P.G.K. has received speaking and consultancy honoraria from Abbvie, Ferring, Janssen, Pfizer and Takeda. S.G. has served as Steering Committee member to Pfizer, Abbvie, Janssen, Aerpio, Receptos, Takeda, Bristol Myers Squibb, Novo Nordisk, Shire, Ferring and has received research support from Abbvie and speaker fee from Abbvie, Takeda, Falk Pharma and Janssen. W.A.B. has received speaking and consultancy honoraria from Abbvie, Covidien and Ethicon (Johnson and Johnson). R.P. has received research/educational support from AbbVie, Abbott, Ferring, Janssen, Schering-Plough, Centocor, Millennium, Elan, Procter \& Gamble, and Bristol-Myers Squibb. He has served as a consultant for AbbVie, Abbott, Amgen, Aptalis, AstraZeneca, Baxter, Eisai, Ferring, Janssen, Merck, Schering-Plough, Shire, Centocor, Elan, GlaxoSmithKline, UCB, Pfizer, Bristol-Myers Squibb, Warner Chilcott, Takeda, Cubist, Celgene, Gilead Sciences, and Takeda. R.P. has also participated on speaker's bureaus for AbbVie, AstraZeneca, Janssen, Schering-Plough, Shire, Ferring, Centocor, Elan, Prometheus, Warner Chilcott, and Takeda. He has attended Advisory Boards for AbbVie, Abbott, Amgen, Aptalis, AstraZeneca, Baxter, Eisai, Ferring, Genentech, Janssen, Merck, ScheringPlough, Shire, Centocor, Elan, GlaxoSmithKline, UCB, Pfizer, Bristol-Myers Squibb, Warner Chilcott, Takeda, Cubist, Celgene, and Salix.

\footnotetext{
(c) Copyright 2017. Korean Association for the Study of Intestinal Diseases. All rights reserved.
}

This is an Open Access article distributed under the terms of the Creative Commons Attribution Non-Commercial License (http://creativecommons.org/licenses/by-nc/4.0)

which permits unrestricted non-commercial use, distribution, and reproduction in any medium, provided the original work is properly cited. 
whether anti-tumor necrosis factor (anti-TNF) therapy in the preoperative period could be a risk factor for both infectious and surgical complications in these patients. Different studies and meta-analyses revealed opposite conclusions, with limited but similar methodological issues. ${ }^{5,6}$ Two prospective cohort studies reported that there can be an increase in postoperative complications after abdominal surgery for CD in patients previously exposed to infliximab (IFX). ${ }^{7,8}$ In one of these studies, Lau et al. ${ }^{7}$ described that higher serum levels of the drug in the preoperative period $(\geq 8 \mu \mathrm{g} / \mathrm{mL})$ were associated with higher readmission and overall complication rates. More prospective data in this field are needed.

Indeed, CD can be heterogeneous, and several phenotypes of the disease can be observed concurrently in clinical practice. Frequently, patients with an established surgical indication for stenosis or internal fistula, for instance, may present with a high inflammatory burden associated to luminal CD or mesenteric masses without abscesses, and need a decrease in their inflammatory status to be optimized for surgery.

The strategy of intentional preoperative anti-TNF therapy in $\mathrm{CD}$ is attractive, and may be part of the real-life practice for severe cases of $\mathrm{CD}$ worldwide. In the management of rectal cancer, neoadjuvant therapy leads to a reduction in the tumor size, with postponed surgery having better outcomes. There is a controversy whether a similar principle could be applied in the management of specific complicated cases of abdominal CD. The question arises whether patients with an established surgical indication, with inflammatory masses, and without infection would receive any benefit from reducing the inflammatory burden with a fast-acting treatment with anti-TNF therapy. Whether the surgical procedure would be facilitated, with the advantages of reduced bleeding, more economic resections, and reduced operative time, still needs to be proven with specific studies. On the contrary, there is the obvious concern about the safety of antiTNF agents in the perioperative period, as previously stated.

The aim of this critical review was to analyze the possible indications, contraindications, and consequences of the intentional use of preoperative anti-TNF therapy in abdominal $\mathrm{CD}$, and to outline the potential variables involved in this strategy.

\section{POSSIBLE INDICATIONS FOR INTENTIONAL PREOPERATIVE ANTI-TNF THERAPY}

Patients with a high inflammatory burden and clear surgical indication could possibly benefit from the strategy of preoperative anti-TNF agents. This would include patients with inflammatory masses in the mesentery or mesocolon, without abscesses or phlegmons, and with associated fibrotic stenosis or internal fistulas. It is known that patients can present different phenotypes of the disease simultaneously. A typical example is the occurrence of an ileal fibrotic stenosis, with proximal dilatation, associated ileitis, and an ileosigmoid fistula. In the same scenario, patients with luminal, stenosing, and penetrating disease, with a clear indication for a surgical procedure, could possibly benefit from a reduction in inflammation with biological agents.

Another possible indication of this strategy is extensive luminal small bowel disease, with specific localized fibrotic stenosis in certain parts of the jejunum and ileum. Preoperative medication could theoretically reduce the inflammatory lesions, leading to mucosal healing, with surgery being indicated as a complementary approach in the fibrotic stenotic areas, with resection or strictureplasty. A third possible indication could be pancolitis associated with $\mathrm{CD}$, in which preoperative anti-TNF could heal parts of the mucosa in determined segments of the large bowel, leading to a change in the surgical indication from a total colectomy to segmental colectomies. Likewise, rectal preservation could be aimed in patients with rectal mucosal healing due to anti-TNF therapy. This can preserve the rectum after the administration of biologics, in fibrotic colons with complete loss of haustrations, and impaired absorption and reservoir function, with ileorectal anastomosis being performed instead of total proctocolectomies. In these cases, previous anti-TNF agents could prevent permanent stomas with preservation of the rectum - certainly a more attractive option to patientswhen properly indicated. In all previously discussed situations (detailed in Table 1), active infection must be excluded.

\section{CONTRAINDICATIONS FOR INTENTIONAL PREOPERATIVE ANTI-TNF THERAPY}

Some patients have absolute contraindications for the strategy of intentional use of anti-TNFs before surgery. ${ }^{9}$ This would obviously include those with concomitant intra- or extra-abdominal infections at the time of the surgical indication. Patients with single or multiple abdominal abscesses would also be included in this group. Moreover, patients with associated systemic infections such as pneumonia, urinary tract infections, or sepsis from any other source clearly are not candidates for this strategy, owing to the significant risk of immunosuppression in this specific population.

Patients with contraindications for the use of anti-TNFs 
Table 1. Main Theoretical Objectives and Indications of Intentional Preoperative Use of Anti-TNF Agents

Possible arguments for intentional preoperative anti-TNF therapy in CD

1. Reduction of inflammatory masses aiming more economic small bowel resections

2. Reduction of inflammation in extensive small bowel disease before strictureplasties or segmental resections

3. Reduction of colonic inflammation that can lead to segmental instead of total colectomies in Crohn's colitis

4. Rectal preservation in patients with chronic refractory colitis with lead pipe colon after rectal mucosal healing, avoiding permanent stomas

5. Optimizing the patient's condition for surgery (reduction of inflammatory burden and improvement in nutritional status through reduction in disease activity)

TNF, tumor necrosis factor.

Table 2. Contraindications for Preoperative Anti-TNF Therapy Owing to Safety Issues

Contraindications for deliberative preoperative anti-TNF therapy in CD

1. Associated abdominal abscess to CD (abdominal infections)

2. Extra-abdominal concomitant infections (pneumonia, urinary tract infection, etc.)

3. Contraindications for anti-TNF therapy per se (demyelinating diseases, heart failure)

4. Emergency situations (bowel perforations, acute or subacute obstructions in the small or large bowel)

TNF, tumor necrosis factor.

in general also cannot benefit from this strategy, and this includes patients with demyelinating disease (multiple sclerosis, multifocal motor neuropathy) and those with American Heart Association grades III and IV heart failure. ${ }^{9}$

Patients with a clear indication for emergency surgery also have contraindications to the preoperative anti-TNF strategy, as the time needed for a response is not available in this situation..$^{10}$ This would include patients with blocked or free perforations in any bowel segment, as well as patients with acute bowel obstruction. These patients need immediate surgical therapy, and cannot be ideally optimized for the previously discussed preoperative strategy (Table 2).

\section{PROMISING THEORETICAL ADVANTAGES OF INTENTIONAL PREOPERATIVE ANTI-TNF THERAPY}

From the surgical perspective, a reduction in an inflammatory mesenteric mass could lead to a less complicated procedure. Usually, the thickened mesentery in CD can be challenging to deal with, and hemostasis often needs achievement of continuous needle suturing owing to considerable tissue friability. Moreover, local edema can also lead to increased surgical time due to technical difficulties and bleeding. Operating tissues in a better condition (with less inflammation) could be a consequence of the intentional preoperative anti-TNF strategy.
A reduction in the inflammatory burden could also change the approach of the procedure. Commonly, large inflammatory masses with associated external or internal fistulas are primarily treated with an open approach with longer incisions and more significant surgical trauma, the consequences of which can influence the postoperative recovery. A reduction in the size of the specimen, including the resected bowel and mesentery, could also make a minimally invasive procedure feasible, and this could be another surgical benefit of preoperative anti-TNFs. Moreover, increased reduction in the complexity of the procedure may possibly lower the need for blood transfusions-a known risk factor for a more complicated postoperative period.

From the clinician's perspective, several benefits could be aimed. First, preoperative anti-TNF with consequent reduction of the inflammatory burden could optimize the patients' status for surgery. This could result in an improvement in nutrition and compensation for anemia, which could decrease the postoperative complication rates. Another important point is the possibility of more economic resections, reducing the chances of intestinal failure in the long term, mainly in patients with previous resections. Although there is lack of evidence on this topic, a reduction in inflammation could limit the resections, mainly in the small bowel, and a negative nutritional impact in the postoperative follow-up could be possibly avoided. Preservation of the rectum can also be a potential advantage from the clinical point of view, 
as a better bowel function can be observed after surgery in cases with ileorectal anastomosis. Stoma avoidance could also be an advantage, as the presence of a stoma has psychological effects on and difficult to accept for patients, in addition to requiring specialized care.

Coscia et al. ${ }^{11}$ described the profile of surgical therapy for colonic CD before and after the biological era. In their retrospective cohort study from Italy, the authors showed that the rate of permanent stomas was $60.8 \%$ in the prebiological era and $19.2 \%$ after this period, when biologics were widely used $(P<0.001)$. Moreover, univariate and multivariate analyses showed that only the use of biologics was significantly associated with an increased rate of rectal preservation in those patients $(P<0.05)$. The preoperative use of anti-TNF agents in these patients did not have an intentional protocol; however, it showed the capacity for rectal preservation possibly owing to mucosal healing caused by the biological agents, which possibly influenced the long-term surgical outcomes.

de Groof et al., ${ }^{12}$ in an interesting study from the Netherlands, compared the length of specimens from ileocolic resections between patients from a population-based cohort and patients from a referral center who had more intensive medical therapy. When grouped according to anti-TNF use, a median length of resected ileum of $21.0 \mathrm{~cm}$ (IQR, 12.533.5) was observed in patients with previous anti-TNF as compared with $20.0 \mathrm{~cm}$ (IQR, 12.0-30.0) in patients without anti-TNF treatment $(P=0.87)$. Patients treated with a more intensive therapy, such as biologics, had a significant delay in the surgical indication from the diagnosis to the first ileocolic resection. This retrospective analysis suggests that the use of anti-TNF agents before surgery still did not result in a reduction in the length of specimens after ileocolic resection, which demonstrates the controversy about the real impact of biologics in bowel sparing.

\section{PERIOPERATIVE ANTI-TNF AND COMPLICATIONS: CURRENT EVIDENCE AND POSSIBLE PITFALLS}

The literature shows that the impact of anti-TNF agents on postoperative outcomes is largely controversial. Several meta-analyses demonstrated different results. ${ }^{5,6}$ In the same way, animal experimental studies were performed and demonstrated opposite results. ${ }^{13,14}$

There are several case series describing the impact of preoperative anti-TNF agents on postoperative outcomes after abdominal procedures in CD. A more detailed analysis of these previous reports shows some differences in the methodology used among studies. Some studies included patients with CD and those with UC in the same sample. ${ }^{15,16}$ The outcomes analyzed were also different among the studies. Some exclusively analyzed surgical complications, others infectious complications, and some postoperative complications in general. ${ }^{5,6,15,16}$ The heterogeneity of these results demonstrates the controversy about the consequences of biological therapy in surgical patients with $\mathrm{CD}$, and this needs to be taken into account if the intentional preoperative antiTNF strategy is aimed.

Only two prospective studies have analyzed the relation between preoperative exposure to anti-TNF therapy and surgical complications in CD. Lau et al. ${ }^{7}$ analyzed 123 patients in whom serum levels of IFX were measured 7 days before the surgical procedure. More overall postoperative complications and more readmissions were observed in patients with IFX levels $>8 \mu \mathrm{g} / \mathrm{mL}$ than in those with levels $<3 \mu \mathrm{g} / \mathrm{mL}$. This difference was only observed in patients with CD, not after surgical procedures in patients with UC. The authors suggested that circulating anti-TNF agents may have an influence on surgical complications.

Similar results were observed in a prospective multicenter French study (RICCO registry), presented at the European Crohn's and Colitis Organisation Congress, 2016. ${ }^{8}$ From a total of 592 patients submitted exclusively to ileocecal resections for $\mathrm{CD}, 23.1 \%$ received previous anti-TNF therapy up to 6 months before surgery. There were higher rates of overall morbidity ( $41 \%$ vs. $26 \%, P=0.001$ ) and abdominal sepsis ( $13 \%$ vs. $7.1 \%, P=0.030)$ in the group of patients with previous anti-TNF than in controls. The use of anti-TNF agents $<6$ months before surgery remained a strong predictor of overall postoperative morbidity (OR, 2.10; 95\% CI, 1.60-2.75; $P<0.0001)$ and intra-abdominal sepsis (OR, 3.13; 95\% CI, $2.07-4.73 ; P<0.001)$. The results of this trial were not based on serum levels. Moreover, as it included patients with exposure to anti-TNF agents up to 6 months before the operations, some patients even could not have any circulating antiTNF agents during the procedures. This raises the question of whether the anti-TNF agent per se was the direct cause of more complications, or if a coincidental profile and more complicated patients were included in the analysis.

Therefore, more prospective comparative data aiming to analyze the effect of preoperative anti-TNF therapy on postoperative surgical outcomes in CD are needed. It has been clearly demonstrated that preoperative steroids and malnutrition increase the complication rates after surgery in $\mathrm{CD} .^{10}$ Usually, sicker patients are treated with biological agents, and this can be the confounding factor resulting in increased postoperative complications in some studies. 


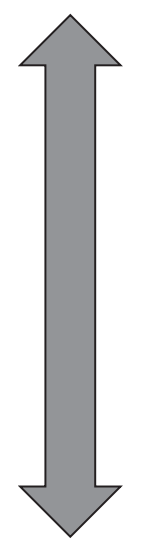

Possible disadvantages

1. Possible increase in postoperative surgical and infectious complications

2. Increase in the cost of care

3. Infections between anti-TNF therapy induction and surgery

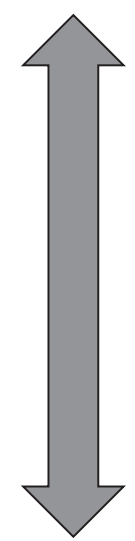

Fig. 1. Balance between possible theoretical advantages and disadvantages of intentional preoperative anti-tumor necrosis factor (anti-TNF) therapy in patients with CD.
As most of the retrospective studies tend to show a lack of influence of these agents on postoperative complications, several referral IBD centers do not routinely interrupt therapy in patients with CD undergoing elective abdominal surgery, which can lead to the rationale of the intentional preoperative strategy described in this critical review. Pitfalls such as preoperative steroid use and malnutrition are also important in this individualized decision.

Usually, biological agents need some time before they exert their full action, and thus before the treatment response can be evaluated. The induction period varies depending on the agent (usually 4 weeks for adalimumab and certolizumab pegol, and 14 weeks for IFX). However, there is expert consensus that at least 12 weeks are needed for patients to acquire the benefit of reduced inflammatory burden. Clearly, infections could occur in this intermediate period (between the first dose of the anti-TNF agent and surgery), based on the previous wide experience with all anti-TNF agents. Therefore, close monitoring is recommended if the intentional preoperative anti-TNF strategy is performed.

Another potential disadvantage of using anti-TNFs intentionally is the obvious significant increase in the therapy costs. ${ }^{17}$ Even with the possibility of reduced surgical time and hospital stay, pharmacoeconomic studies would be needed to better clarify the cost-benefit ratio of this strategy. The potential theoretical advantages and disadvantages of intentional preoperative use of anti-TNF agents are described in Fig. 1.

\section{FINAL MESSAGES AND CONCLUSIONS}

Deliberative use of preoperative anti-TNF therapy in patients with CD can be challenging and it requires more research for implementation in clinical practice. The potential benefits need to be specifically balanced against the possible risks of increasing postoperative complication rates, as there is controversy in the literature about whether anti-TNF treatment worsens outcomes because of the agents per se or as a coincidental factor related to the more severe disease of patients. Thus, the decision to intentionally administer antiTNF before surgery must be individualized and be made by a multidisciplinary team, including surgeons and gastroenterologists, to optimize the outcomes in patients specifically selected for this strategy. Further studies are urgently needed. However, in the meantime, the authors believe that on the balance of evidence, there is a place for intentional preoperative anti-TNF therapy in very carefully selected patients.

\section{ACKNOWLEDGEMENTS}

P.G.K., S.G., W.A.B., and R.P. drafted the manuscript and gave equally important intellectual content.

\section{REFERENCES}

1. Danese S. New therapies for inflammatory bowel disease: from the bench to the bedside. Gut 2012;61:918-932.

2. Maggiori L, Panis Y. Laparoscopy in Crohn's disease. Best Pract Res Clin Gastroenterol 2014;28:183-194.

3. Spinelli A, Bazzi P, Sacchi M, et al. Short-term outcomes of laparoscopy combined with enhanced recovery pathway after ileocecal resection for Crohn's disease: a case-matched analysis. J Gastrointest Surg 2013;17:126-132.

4. de Buck van Overstraeten A, Wolthuis A, D'Hoore A. Surgery for Crohn's disease in the era of biologicals: a reduced need or delayed verdict? World J Gastroenterol 2012;18:3828-3832.

5. Narula N, Charleton D, Marshall JK. Meta-analysis: peri-operative anti-TNFalpha treatment and post-operative complications in patients with inflammatory bowel disease. Aliment Pharmacol Ther 2013;37:1057-1064. 
6. Billioud V, Ford AC, Tedesco ED, Colombel JF, Roblin X, PeyrinBiroulet L. Preoperative use of anti-TNF therapy and postoperative complications in inflammatory bowel diseases: a metaanalysis. J Crohns Colitis 2013;7:853-867.

7. Lau C, Dubinsky M, Melmed G, et al. The impact of preoperative serum anti-TNFalpha therapy levels on early postoperative outcomes in inflammatory bowel disease surgery. Ann Surg 2015;261:487-496.

8. Brouquet A, Maggiori L, Zerbib P, et al. Anti-TNF therapy is associated with increased risk of postoperative morbidity after surgery for ileocolonic Crohn disease: outcome analysis in a prospective nationwide cohort of 592 patients conducted by the GETAID chirurgie group. J Crohns Colitis 2016;10(Suppl 1):S22-S23.

9. D'Haens GR, Panaccione R, Higgins PD, et al. The London Position Statement of the World Congress of Gastroenterology on Biological Therapy for IBD with the European Crohn's and Colitis Organization: when to start, when to stop, which drug to choose, and how to predict response? Am J Gastroenterol 2011;106:199-212.

10. Schlussel AT, Steele SR, Alavi K. Current challenges in the surgical management of Crohn's disease: a systematic review. Am J Surg 2016;212:345-351.

11. Coscia M, Gentilini L, Laureti S, et al. Risk of permanent stoma in extensive Crohn's colitis: the impact of biological drugs. Colorectal Dis 2013;15:1115-1122.
12. de Groof EJ, Gardenbroek TJ, Buskens CJ, et al. The association between intensified medical treatment, time to surgery and ileocolic specimen length in Crohn's disease [published online ahead of print November 24, 2016]. Colorectal Dis. doi: 10.1111/codi.13567.

13. Frostberg E, Ström P, Gerke O, Qvist N. Infliximab's influence on anastomotic strength and degree of inflammation in intestinal surgery in a rabbit model. BMC Surg 2014;14:23.

14. Jensen JS, Petersen NB, Biagini M, Bollen P, Qvist N. Infliximab treatment reduces tensile strength in intestinal anastomosis. J Surg Res 2015;193:145-152.

15. Waterman M, Xu W, Dinani A, et al. Preoperative biological therapy and short-term outcomes of abdominal surgery in patients with inflammatory bowel disease. Gut 2013;62:387-394.

16. Appau KA, Fazio VW, Shen B, et al. Use of infliximab within 3 months of ileocolonic resection is associated with adverse postoperative outcomes in Crohn's patients. J Gastrointest Surg 2008;12:1738-1744.

17. Marchetti M, Liberato NL. Biological therapies in Crohn's disease: are they cost-effective? A critical appraisal of modelbased analyses. Expert Rev Pharmacoecon Outcomes Res 2014;14:815-824. 